Electronic Journal of Statistics

Vol. 3 (2009) 165-175

ISSN: $1935-7524$

DOI: $10.1214 / 07-E J S 066$

\title{
An exponential inequality for negatively associated random variables
}

\author{
H. Jabbari* and M. Jabbari* \\ Department of Statistics, Ferdowsi University of Mashhad, Mashhad, Iran \\ e-mail: jabbarinh@um.ac.ir; jabbarinm@yahoo.com \\ H.A. Azarnoosh* \\ Department of Statistics, Ferdowsi University of Mashhad, Mashhad, Iran \\ e-mail: azarnush@math.um.ac.ir \\ url: http://mathstat.um.ac.ir
}

\begin{abstract}
We prove an exponential inequality for negatively associated and strictly stationary random variables. A condition is given for almost sure convergence and the associated rate of convergence is specified in terms of the underlying covariance function.
\end{abstract}

AMS 2000 subject classifications: Primary 60F15, 60K35; secondary $62 \mathrm{G} 20$.

Keywords and phrases: Covariance function, Exponential inequality, Negative association.

Received May 2007.

\section{Contents}

1 Introduction . . . . . . . . . . . . . . . . . . . 165

2 Definitions, notations and formulation of main results . . . . . . . 166

3 Preliminary results . . . . . . . . . . . . . . . . . . . 168

4 Examples . . . . . . . . . . . . . . . . . . . . . . . . . 174

Acknowledgements . . . . . . . . . . . . . . . . . . . 174

References . . . . . . . . . . . . . . . . . . . . . . 174

\section{Introduction}

In nonparametric estimation, exponential inequalities of Bernstein type represent a powerful tool for proving convergence rates. The significance of exponential inequalities toward several probability and statistical applications is well known. There exist several versions available in the literature for independent sequences of variables. Sometimes, the random variables are positively associated. Ioannides and Roussas [4] proved an exponential inequality for positively associated random variables under some assumptions of uniform boundedness

* The authors wish to acknowledge partial support from Statistics Center of Excellence of Ferdowsi University of Mashhad. 
and some conditions on the covariance structure of the variables. Oliveira [9] extended these results by dropping the boundedness assumption.

One of dependent structure of random variables (r.v.'s) that has attracted the interest of probabilists and statisticians is negative association (NA). This concept is one qualitative version of negative dependence among random variables. For other versions of negative dependence, such as upper (lower) orthant dependence, reverse regular of order two in pairs, conditionally decreasing in sequence and negatively dependent in sequence, we refer to Lehmann [8], Block et al. [1], Ebrahimi and Ghosh [3], Joag-dev and Patil [6], and Karlin and Rinott [7]. Among those types of negative dependence, only the NA class enjoys the important property of being closed under formation of increasing functions of disjoint sets of random variables. As pointed out and proved by Joag-dev and Proschan [5], a number of well known multivariate distributions possess the NA property, such as (a) multinomial, (b) convolution of unlike multinomial, (c) multivariate hypergeometric, (d) Dirichlet, (e) Dirichlet compound multinomial, (f) negatively correlated normal distribution, (g) permutation distribution, (h) random sampling without replacement, and (i) joint distribution of ranks. Because of their wide applications in multivariate statistical analysis and reliability theory, the notations of negatively associated random variables have received more and more attention recently.

The article is organized as follows: in the next section, the necessary notation and terminology are introduced before the main result. In addition to the basic assumption of negative association, we require that the r.v.'s are uniformly bounded and we impose some further conditions on the covariance structure, see assumptions (A1)-(A3). The proof of the theorem rests on Lemma 3.4 which is formulated and proved in Section 3. Actually, all preliminary results needed are taken care of in the same section, as is the proof of the theorem. The optimal associated rate of convergence depends on the underlying covariance function and is explicitly calculated by way of formulas (3.34) and (3.31) for given covariance

function. In Section 4, we discuss two classes of covariances falling into scope of Theorem 2.1. To avoid unnecessary repetitions, it is stated at the outset that all limits are taken as $n \rightarrow \infty$.

\section{Definitions, notations and formulation of main results}

At first, we introduce two definitions for ND and NA random variables.

Definition 2.1. Two random variables $X$ and $Y$ are negatively quadrant dependent (NQD) if for every $x, y \in R$ we have

$$
\mathbf{P}(X \leq x, Y \leq y) \leq \mathbf{P}(X \leq x) \mathbf{P}(Y \leq y) .
$$

Definition 2.2. A finite family of random variables $\left\{X_{i}, 1 \leq i \leq n\right\}$ is said to be negatively associated (NA) if for every pair of disjoint subsets A and B of $\{1,2, \ldots, n\}$,

$$
\operatorname{Cov}\left(f_{1}\left(X_{i}, i \epsilon A\right), f_{2}\left(X_{j}, j \epsilon B\right)\right) \leq 0 .
$$


whenever $f_{1}$ and $f_{2}$ are coordinatewise increasing and such that the covariance exists. An infinite family of random variables is NA if every finite subfamily is NA.

Consider a sequence of natural numbers $p_{n}$ such that, for each $n \geq 1,1 \leq$ $p_{n}<n$ and $p_{n} \rightarrow \infty$. Then, we divide the set $\{1,2, \ldots, n\}$ into successive groups

each containing $p_{n}$ elements. Define $r_{n}$ as the greatest integer less or equal to $n / 2 p_{n}$, which implies that $n / 2 r_{n} p_{n} \rightarrow 1$. Thus the set $\{1,2, \ldots, n\}$ is split into $2 r_{n}$ groups, each consisting of $p_{n}$ elements; the remaining $n-2 r_{n} p_{n}<p_{n}$ elements constitute a set which may be empty.

For easy reference, we consider some assumptions that the main result in this paper remained valid.

\section{Assumptions}

(A1) The basic assumption is that the r.v.'s $\left\{X_{i}, i \geq 1\right\}$ are NA.

(A2) The r.v.'s are bounded, $|X| \leq M / 2, i \geq 1$ ( $M$ is independent of $i$ ) and covariance invariant,

$$
\operatorname{Cov}\left(X_{i}, X_{i+k}\right)=\operatorname{Cov}\left(X_{1}, X_{k+1}\right) . i \geq 1, k \geq 1
$$

(A3) Without loss of generality, it is assumed $\operatorname{Cov}\left(X_{1}, X_{k+1}\right)$ is nondecreasing as $k \rightarrow \infty$.

Remark 2.1. Covariance invariant in assumption (A2) can be dropped, if we assumed

$$
\operatorname{Cov}\left(X_{1}, X_{k+1}\right)=\inf \left\{\operatorname{Cov}\left(X_{i}, X_{i+k}\right) ; i \geq 1\right\}, k \geq 1 .
$$

Define $\bar{S}_{n}$ and $\varepsilon_{n}$ by

$$
\bar{S}_{n}=\frac{1}{n} \sum_{i=1}^{n}\left(X_{i}-\mathrm{E} X_{i}\right), \quad \varepsilon_{n}=\left(\frac{\alpha M^{2}}{2}\right)^{1 / 2}\left(\frac{\log n}{r_{n}}\right)^{1 / 2},
$$

where $\mathrm{M}$ is as in assumption (A2) and $\alpha$ is an arbitrary constant greater than one. Then the main result obtained in following theorem.

Theorem 2.1. Let $\bar{S}_{n}$ and $\varepsilon_{n}$ be defined by (2.4). Then, under assumptions (A1) and (A2), and the proviso

$$
\operatorname{Cov}\left(X_{1}, X_{k+1}\right) \leq \exp \left\{-\frac{4(M+1)}{3} M\left(\frac{\alpha}{2}\right)^{1 / 2}\left(r_{n} \log n\right)^{1 / 2}\right\},
$$

it holds

$$
P\left(\left|\bar{S}_{n}\right| \geq \varepsilon_{n}\right) \leq C_{0} \exp \left(-c r_{n} \varepsilon_{n}{ }^{2}\right), \quad c=2 / 9 M^{2}
$$

for all sufficiently large $n, n \geq n_{0}$, where $C_{0}$ is a constant.

Furthermore, $\bar{S}_{n} \rightarrow 0$ a.s. at the rate $1 / \varepsilon_{n}$. The optimal specification of $r_{n}$ is given by (3.33) or (3.34) and the respective $1 / \varepsilon_{n}$ is given by (3.31). 


\section{Preliminary results}

Let $Y_{i}=X_{i}-\mathrm{E} X_{i}$, so that $\left|Y_{i}\right| \leq M, i \geq 1$ are NA and $\bar{S}_{n}=n^{-1} \sum_{i=1}^{n} Y_{i}$. Define the r.v.'s $U_{i}, V_{i}, i=1, \ldots, r_{n}$ and $W_{n}$ by

$$
\begin{gathered}
U_{i}=Y_{2(i-1) p_{n}+1}+\cdots+Y_{(2 i-1) p_{n}}, \quad V_{i}=Y_{(2 i-1) p_{n}+1}+\cdots+Y_{2 i p_{n}}, \\
W_{n}=Y_{2 p_{n} r_{n}+1}+\cdots+Y_{n}
\end{gathered}
$$

where $p_{n}$ and $r_{n}$ are as in the previous section and

$$
\bar{U}_{n}=\frac{1}{n} \sum_{i=1}^{r_{n}} U_{i}, \quad \bar{V}_{n}=\frac{1}{n} \sum_{i=1}^{r_{n}} V_{i}, \quad \bar{W}_{n}=\frac{W_{n}}{n} .
$$

So that

$$
\bar{S}_{n}=\bar{U}_{n}+\bar{V}_{n}+\bar{W}_{n}
$$

Lemma 3.1. Suppose $X$ and $Y$ are $N Q D$ random variables with finite variance and $f, g$ are complex valued functions on $R^{1}$ with $f^{\prime}$ and $g^{\prime}$ bounded. Then

$$
|\operatorname{Cov}(f(X), g(Y))| \leq-\left\|f^{\prime}\right\|_{\infty}\left\|g^{\prime}\right\|_{\infty} \operatorname{Cov}(X, Y),
$$

where $\|$.$\| denotes the sup norm on R^{1}$; in particular, for any real s and $t$,

$$
\left|\mathrm{E}\left(e^{i s X+i t Y}\right)-\mathrm{E}\left(e^{i s X}\right) \mathrm{E}\left(e^{i t Y}\right)\right| \leq-|s||t| \operatorname{Cov}(X, Y) .
$$

Proof. Define

$$
\mathrm{H}(x, y)=\mathbf{P}(X \leq x, Y \leq y)-\mathbf{P}(X \leq x) \mathbf{P}(Y \leq y) .
$$

By Hoeffding lemma [8],

$$
\operatorname{Cov}(X, Y)=\int_{-\infty}^{\infty} \int_{-\infty}^{\infty} \mathrm{H}(x, y) d x d y
$$

This equation can be easily generalized (see, for more information Newman, 1980) to yield

$$
\operatorname{Cov}(f(X), g(Y))=\int_{-\infty}^{\infty} \int_{-\infty}^{\infty} f^{\prime}(x) g^{\prime}(y) \mathrm{H}(x, y) d x d y .
$$

Since $X$ and $Y$ are NQD random variables $\mathrm{H}(x, y) \leq 0$, thus

$$
\begin{aligned}
|\operatorname{Cov}(f(X), g(Y))| & =\int_{-\infty}^{\infty} \int_{-\infty}^{\infty}\left|f^{\prime}(x)\right| \cdot\left|g^{\prime}(y)\right||\mathrm{H}(x, y)| d x d y \\
& \leq\left\|f^{\prime}\right\|_{\infty} \cdot\left\|g^{\prime}\right\|_{\infty} \operatorname{Cov}(X, Y)
\end{aligned}
$$

as desired. 
Lemma 3.2. Let $X_{1}, X_{2}, \ldots, X_{n}$ be a sequence of $N A$ random variables bounded by constant $\delta^{\prime}$. Then, for every $\lambda>0$,

$$
\left|\operatorname{Cov}\left(e^{\lambda \sum_{i=1}^{n-1} X_{i}}, e^{\lambda X_{n}}\right)\right| \leq-\lambda^{2} e^{n \lambda \delta^{\prime}} \sum_{1 \leq i<j \leq n} \operatorname{Cov}\left(X_{i}, X_{j}\right) .
$$

Proof. By Lemma 3.1 for $\lambda>0$, we have

$$
\left|\operatorname{Cov}\left(e^{\lambda X_{1}}, e^{\lambda X_{2}}\right)\right| \leq \lambda^{2} e^{2 \lambda \delta^{\prime}} \operatorname{Cov}\left(X_{1}, X_{2}\right) .
$$

The result follows by induction and using the fact that if $X, Y$ and $Z$ are NA then so are $X$ and $Y+Z$ as they are increasing functions of NA r.v.'s.

We quote next a general lemma used to control some of the terms appearing in the course of proof.

Lemma 3.3 ([2]). Let $W$ be a central random variable. If there exist $a, b \in R$ such that $\mathrm{P}(a \leq W \leq b)=1$ then, for every $\lambda>0$

$$
\left|\mathrm{E}\left(e^{\lambda W}\right)\right| \leq \exp \left(\frac{\lambda^{2}(b-a)^{2}}{8}\right) .
$$

Lemma 3.4. Let $\varepsilon_{n}>0$ and $\bar{U}_{n}$ be defined by (3.3) and suppose assumptions (A1)-(A3) hold. Then, for an appropriate constant $C_{0}$,

$$
\mathrm{P}\left(\bar{U}_{n} \geq \varepsilon_{n}\right) \leq C_{0} \exp \left(-2 r_{n} \varepsilon_{n}{ }^{2} / M^{2}\right)
$$

provided

$$
-\operatorname{Cov}\left(X_{1}, X_{k+1}\right) \leq \exp \left(-4(M+1) r_{n} \varepsilon_{n} / M^{2}\right) .
$$

Proof. The r.v.'s $U_{1}, \ldots, U_{r_{n}}$ are NA and $\left|U_{i}\right| \leq p_{n} M$ for all $i$. For some $\lambda>0$, set $h(x)=e^{\frac{\lambda}{n} x},-p_{n} M<x<p_{n} M$ so that $h^{\prime}(x)=\frac{\lambda}{n} e^{\frac{\lambda}{n} x}$ and for their supnorms, it holds: $\|h\|_{\infty} \leq e^{\lambda p_{n} \frac{M}{n}},\left\|h^{\prime}\right\|_{\infty} \leq \frac{\lambda}{n} e^{\lambda p_{n} \frac{M}{n}}$. With this $h$ and $A=$ $\left\{1, \ldots, r_{n}-1\right\}, B=\left\{r_{n}\right\}$, so that $\sharp A+\sharp B-2=r_{n}-2$, apply Lemma 3.2 to obtain

$$
\operatorname{Cov}\left(e^{\frac{\lambda}{n} \sum_{i=1}^{r_{n}-1} U_{i}}, e^{\frac{\lambda}{n} U_{r_{n}}}\right) \leq-\frac{\lambda^{2}}{n^{2}} e^{\lambda p_{n} r_{n} \frac{M}{n}} \sum_{i=1}^{r_{n}-1} \operatorname{Cov}\left(U_{i}, U_{r_{n}}\right),
$$

so that

$$
\begin{aligned}
\mathrm{E}\left(e^{\lambda \bar{U}_{n}}\right) & =\mathrm{E}\left(e^{\frac{\lambda}{n} \sum_{i=1}^{r_{n}-1} U_{i}} \cdot e^{\frac{\lambda}{n} U_{r_{n}}}\right) \\
& \leq \mathrm{E} e^{\frac{\lambda}{n} \sum_{i=1}^{r_{n}-1} U_{i}} \mathrm{E} e^{\frac{\lambda}{n} U_{r_{n}}}-\frac{\lambda^{2}}{n^{2}} e^{\lambda p_{n} r_{n} \frac{M}{n}} \sum_{i=1}^{r_{n}-1} \operatorname{Cov}\left(U_{i}, U_{r_{n}}\right) .
\end{aligned}
$$


However

$$
\begin{aligned}
\sum_{i=1}^{r_{n}-1} \operatorname{Cov}\left(U_{i}, U_{r_{n}}\right) & =\sum_{i=1}^{r_{n}-1} \sum_{j=2(i-1) p_{n}+1}^{(2 i-1) p_{n}} \sum_{k=2\left(r_{n}-1\right) p_{n}+1}^{\left(2 r_{n}-1\right) p_{n}} \operatorname{Cov}\left(Y_{j}, Y_{k}\right) \\
& \leq \sum_{j \in A} \sum_{k \in B} \operatorname{Cov}\left(Y_{j}, Y_{k}\right) \\
& =\sum_{i=1}^{r_{n}-1} \sum_{j \in A_{i}} \sum_{k \in B} \operatorname{Cov}\left(Y_{j}, Y_{k}\right)
\end{aligned}
$$

where

$$
\begin{aligned}
& \mathrm{A}=\{\underbrace{1, \ldots, p_{n}} ; \underbrace{2 p_{n}+1, \ldots, 3 p_{n}} ; \ldots ; \underbrace{2\left(r_{n}-2\right) p_{n}+1, \ldots,\left(2 r_{n}-3\right) p_{n}}\} \\
& =\left\{A_{1} ; A_{2} ; \ldots ; A_{r_{n}-1}\right\}
\end{aligned}
$$

and $\mathrm{B}=\left\{2\left(r_{n}-2\right) p_{n}+1, \ldots,\left(2 r_{n}-3\right) p_{n}\right\}$. By assumptions (A2) and (A3),

$$
\begin{aligned}
\sum_{j \in A_{1}} \sum_{k \in B} \operatorname{Cov}\left(Y_{j}, Y_{k}\right) \geq & p_{n} \operatorname{Cov}\left(Y_{1}, Y_{2\left(r_{n}-1\right) p_{n}+1}\right)+\cdots \\
& +p_{n} \operatorname{Cov}\left(Y_{p_{n}}, Y_{2\left(r_{n}-1\right) p_{n}+1}\right) \\
\geq & p_{n}^{2} \operatorname{Cov}\left(Y_{p_{n}}, Y_{\left.2\left(r_{n}-1\right) p_{n}+1\right)}\right.
\end{aligned}
$$

Similarly,

$$
\sum_{j \in A_{2}} \sum_{k \in B} \operatorname{Cov}\left(Y_{j}, Y_{k}\right) \geq p_{n}^{2} \operatorname{Cov}\left(Y_{p_{n}}, Y_{2\left(r_{n}-2\right) p_{n}+1}\right)
$$

and continuing like this

$$
\sum_{j \in A_{r_{n}-1}} \sum_{k \in B} \operatorname{Cov}\left(Y_{j}, Y_{k}\right) \geq p_{n}^{2} \operatorname{Cov}\left(Y_{p_{n}}, Y_{2 p_{n}+1}\right) .
$$

Thus,

$$
\begin{aligned}
\sum_{j \in A} \sum_{k \in B} \operatorname{Cov}\left(Y_{j}, Y_{k}\right) \geq & p_{n}^{2}\left[\operatorname{Cov}\left(Y_{p_{n}}, Y_{2 p_{n}+1}\right)+\cdots+\operatorname{Cov}\left(Y_{p_{n}}, Y_{2\left(r_{n}-2\right) p_{n}+1}\right)\right. \\
& +\operatorname{Cov}\left(Y_{p_{n}}, Y_{\left.2\left(r_{n}-1\right) p_{n}+1\right)}\right) \\
\geq & p_{n}^{2} r_{n} \operatorname{Cov}\left(Y_{p_{n}}, Y_{2 p_{n}+1}\right) .
\end{aligned}
$$

By means of (3.15) and (3.14), inequality (19) becomes

$\mathrm{E}\left(e^{\lambda \bar{U}_{n}}\right) \leq \mathrm{E} e^{\frac{\lambda}{n} \sum_{i=1}^{r_{n}-1} U_{i}} \mathrm{E} e^{\frac{\lambda}{n} U_{r_{n}}}-\frac{\lambda^{2}}{n^{2}} e^{\lambda p_{n} r_{n} \frac{M}{n}} p_{n}^{2} r_{n} \operatorname{Cov}\left(Y_{p_{n}}, Y_{2 p_{n}+1}\right)$.

By applying the inequality $1+x \leq e^{x}, x \in R$, for $x=\frac{\lambda}{n} U_{r_{n}}$ and $x=\frac{\lambda}{n} \sum_{i=1}^{r_{n}-1} U_{i}$ and getting expectations, we have

$$
1 \leq \mathrm{E} e^{\frac{\lambda}{n} \sum_{i=1}^{r_{n}-1} U_{i}} \mathrm{E}^{\frac{\lambda}{n} U_{r_{n}}} .
$$


From $2 p_{n} r_{n} \leq n$, we get $\frac{p_{n}^{2} r_{n}}{n^{2}} \leq 1 / 4 r_{n}$. Therefore, inequality (3.16) becomes

$$
\mathrm{E}\left(e^{\lambda \bar{U}_{n}}\right) \leq \mathrm{E} e^{\frac{\lambda}{n} \sum_{i=1}^{r_{n}-1} U_{i}} \mathrm{E}^{\frac{\lambda}{n} U_{r_{n}}}\left[1-\frac{\lambda^{2}}{4 r_{n}} e^{\lambda M} \operatorname{Cov}\left(Y_{p_{n}}, Y_{2 p_{n}+1}\right)\right] .
$$

The inequality $x e \leq e^{x}, x \in R$ gives $\frac{\lambda^{2}}{4} \leq e^{-2} e^{\lambda}<e^{\lambda}$, so that

$$
1-\frac{\lambda^{2}}{4 r_{n}} e^{\lambda M} \operatorname{Cov}\left(Y_{p_{n}}, Y_{2 p_{n}+1}\right) \leq 1-\frac{1}{r_{n}} e^{\lambda(1+M)} \operatorname{Cov}\left(Y_{p_{n}}, Y_{2 p_{n}+1}\right),
$$

and we wish to have $-e^{\lambda(1+M)} \operatorname{Cov}\left(Y_{p_{n}}, Y_{2 p_{n}+1}\right) \leq 1$ or

$$
\lambda \leq-\frac{1}{M+1} \log \left(-\operatorname{Cov}\left(Y_{p_{n}}, Y_{2 p_{n}+1}\right)\right) .
$$

On account of (3.19) and (3.20), inequality (3.18) yields

$$
\mathrm{E}\left(e^{\lambda \bar{U}_{n}}\right) \leq \mathrm{E} e^{\frac{\lambda}{n} \sum_{i=1}^{r_{n}-1} U_{i}} \mathrm{E} e^{\frac{\lambda}{n} U_{r_{n}}}\left[1+\frac{1}{r_{n}}\right] .
$$

Repeating the process which led to (3.21) another $r_{n}-1$ times, we obtain, under condition (3.20),

$$
\mathrm{E}\left(e^{\lambda \bar{U}_{n}}\right) \leq\left[1+\frac{1}{r_{n}}\right]^{r_{n}} \prod_{i=1}^{r_{n}} \mathrm{E} e^{\frac{\lambda}{n} U_{i}}
$$

By applying Lemma 3.3 and taking $W=U_{i}$, we have $\left|U_{i}\right| \leq p_{n} M$ and $b-a=$ $2 p_{n} M$. Then

$$
\prod_{i=1}^{r_{n}} \mathrm{E} e^{\frac{\lambda}{n} U_{i}} \leq e^{\lambda^{2} M^{2} \frac{p_{n}^{2} r_{n}}{2 n^{2}}} \leq e^{\frac{\lambda^{2} M^{2}}{8 r_{n}}} .
$$

Since also $\left(1+\frac{1}{r_{n}}\right)^{r_{n}} \leq C_{1}$ for any positive constant $C_{1}$, inequality (3.22) becomes

$$
\mathrm{E} e^{\lambda \bar{U}_{n}} \leq C_{1} e^{\frac{\lambda^{2} M^{2}}{8 r_{n}}} \text {. subject to (3.20) }
$$

Thus, for $\varepsilon_{n}>0$ and under (3.20)

$$
\mathrm{P}\left(\bar{U}_{n} \geq \varepsilon_{n}\right) \leq C_{1} \exp \left(-\lambda \varepsilon_{n}+\frac{\lambda^{2} M^{2}}{8 r_{n}}\right) .
$$

By minimizing the right-hand side in (3.25) with respect to $\lambda$, we have

$$
\mathrm{P}\left(\bar{U}_{n} \geq \varepsilon_{n}\right) \leq C_{1} \exp \left(-2 \frac{r_{n} \varepsilon^{2}{ }_{n}}{M^{2}}\right),
$$

for $\lambda_{0}=4 \frac{r_{n} \varepsilon_{n}}{M^{2}}$ subject to (3.20).

For $\lambda_{0}$ as in (3.26), (3.20) is equivalent to

$$
-\operatorname{Cov}\left(Y_{p_{n}}, Y_{2 p_{n}+1}\right) \leq \exp \left\{-\frac{4(M+1)}{M^{2}} r_{n} \varepsilon_{n}\right\} .
$$

This completes the proof of the lemma. 
Remark 3.1. As stated in assumption (A3), the condition that the covariance function $\operatorname{Cov}\left(X_{1}, X_{k+1}\right)$ be nondecreasing is not, really, necessary, although it would not be easy to envision cases where it does not occur. This can be justified in the process of arriving at inequality (3.15) by way of (3.14). All one has to do is to produce some more refined bounds for the covariances, but such a result does not appear worth the effort.

For almost sure convergence purposes, we wish to have $2 \frac{r_{n} \varepsilon_{n}^{2}}{M^{2}}=\log n^{\alpha}$ (for any arbitrary $\alpha>1$ ), or equivalently,

$$
\varepsilon_{n}=\left(\frac{\alpha M^{2}}{2}\right)^{1 / 2}\left(\frac{\log n}{r_{n}}\right)^{1 / 2}
$$

Then, $\lambda_{0}$ becomes

$$
\lambda_{0}=\left(\frac{8 \alpha}{M^{2}}\right)^{1 / 2}\left(r_{n} \log n\right)^{1 / 2}
$$

and condition (3.27) yields

$$
-\operatorname{Cov}\left(Y_{p_{n}}, Y_{2 p_{n}+1}\right) \leq \exp \left\{-\frac{4(M+1)}{M}\left(\frac{\alpha}{2}\right)^{1 / 2}\left(r_{n} \log n\right)^{1 / 2}\right\} .
$$

The following lemma summarizes these results.

Lemma 3.5. Suppose assumptions (A1)-(A3) hold. With $\varepsilon_{n}$ specified by (3.28), we have

$$
\mathrm{P}\left(\bar{U}_{n} \geq \varepsilon_{n}\right) \leq C_{1} \exp \left(-2 \frac{r_{r} \varepsilon_{n}^{2}}{M^{2}}\right),
$$

provided $\operatorname{Cov}\left(Y_{p_{r}}, Y_{2 p_{r}+1}\right)$ satisfies condition (3.30).

Remark 3.2. It is obvious that $\bar{V}_{n}$, as defined in (3.3), satisfies the same inequalities as $\bar{U}_{n}$ in Lemmas 3.4 and 3.5.

We may now dispense $\bar{W}_{n}$ as defined in (3.3).

Lemma 3.6. Under assumptions (A1)-(A3) and with $\varepsilon_{n}$ defined by (3.28), $\mathrm{P}_{\mathrm{r}}\left(\left|\bar{W}_{n}\right| \geq \varepsilon_{n}\right)=0$ for any large enough $n$.

Proof. $W_{n}$ consists of $n-2 p_{n} r_{n}$ terms and $n-2 p_{n} r_{n}<p_{n}$. Then, $\left|\bar{W}_{n}\right|<$ $p_{n} \frac{M}{n}=0$. So that $\mathrm{P}\left(\left|\bar{W}_{n}\right| \geq \varepsilon_{n}\right) \leq \mathrm{P}\left(M \geq \frac{n \varepsilon_{n}}{p_{n}}\right)$. However, for any large enough $n$, this last expression is 0 .

Proof of Theorem 2.1. From Lemma 3.5, Remark 3.2 and Lemma 3.6, we obtain that the r.v.'s $-Y_{i}, i=1,2, \ldots, n$ have the same properties as the r.v.'s $Y_{i}$, $i=1,2, \ldots, n$. Thus, under condition (3.30) we have

$$
\mathrm{P}\left(\left|\bar{U}_{n}\right| \geq \varepsilon_{n}\right)=\mathrm{P}\left(\bar{U}_{n} \geq \varepsilon_{n}\right)+\mathrm{P}\left(-\bar{U}_{n} \geq \varepsilon_{n}\right) \leq 2 C_{0} \exp \left(-2 \frac{r_{n} \varepsilon_{n}^{2}}{M^{2}}\right),
$$


and similarly for $\mathrm{P}_{\mathrm{r}}\left(\left|\bar{V}_{n}\right| \geq \varepsilon_{n}\right)$. Then,

$$
\begin{aligned}
\mathrm{P}\left(\left|\bar{S}_{n}\right| \geq 3 \varepsilon_{n}\right) & \leq \mathrm{P}\left(\left|\bar{U}_{n}\right| \geq \varepsilon_{n}\right)+\mathrm{P}\left(\left|\bar{V}_{n}\right| \geq \varepsilon_{n}\right)+\mathrm{P}\left(\left|\bar{W}_{n}\right| \geq \varepsilon_{n}\right) \\
& \leq \mathrm{P}\left(\left|\bar{U}_{n}\right| \geq \varepsilon_{n}\right)+\mathrm{P}\left(\left|\bar{V}_{n}\right| \geq \varepsilon_{n}\right) \quad\left(\text { for } n \geq n_{0}, \text { say }\right) \\
& \leq 4 C_{1} \exp \left(-2 \frac{r_{n} \varepsilon_{n}^{2}}{M^{2}}\right) .
\end{aligned}
$$

Finally, we obtain

$$
\mathrm{P}\left(\left|\bar{S}_{n}\right| \geq \varepsilon_{n}\right) \leq C_{0} \exp \left(-c r_{n} \varepsilon_{n}^{2}\right), \quad c=2 / 9 M^{2}, \quad n \geq n_{0},
$$

where $C_{0}=4 C_{1}$ provided

$$
-\operatorname{Cov}\left(Y_{p_{n}}, Y_{2 p_{n}+1}\right) \leq \exp \left\{-\frac{4(M+1)}{3 M^{2}}\left(\frac{\alpha}{2}\right)^{1 / 2}\left(r_{n} \log n\right)^{1 / 2}\right\} .
$$

Then, $\bar{S}_{n}$ convergence to zero a.s. at the rate of $1 / \varepsilon_{n}$.

For the value of $n$ specified in (3.28), the rate of convergence is given by

$$
\frac{1}{\varepsilon_{n}}=\left(\frac{2}{\alpha M^{2}}\right)^{1 / 2}\left(\frac{r_{n}}{\log n}\right)^{1 / 2} \text {. }
$$

Inequality (3.30) becomes, equivalently:

$$
r_{n} \leq \frac{1}{8 \alpha}\left(\frac{M}{M+1}\right)^{2} \frac{\log ^{2}\left(-\operatorname{Cov}\left(X_{p_{n}}, X_{2 p_{n}+1}\right)\right)}{\log n} .
$$

Expression (3.31) shows that the maximum rate is attained for the maximum allowed value of $r_{n}$. This maximum value is obtained from (3.32) and is

$$
r_{n}=\frac{1}{8 \alpha}\left(\frac{M}{M+1}\right)^{2} \frac{\log ^{2}\left(-\operatorname{Cov}\left(X_{p_{n}}, X_{2 p_{n}+1}\right)\right)}{\log n} .
$$

This is so, because when $r_{n}$ increases, $p_{n}$ decreases, due to the fact that $r_{n}$ and $p_{n}$ are inverse proportional and also to the assumption that $\operatorname{Cov}\left(X_{1}, X_{k+1}\right)$ is an increasing function. Again, by the fact that $n / 2 p_{n} r_{n} \rightarrow 1$, it follows that $p_{n}=\frac{1}{2 x_{n}} \frac{n}{r_{n}}$, some $0<x_{n} \rightarrow 1$. So that (3.31) becomes

$$
\begin{aligned}
& r_{n}=\frac{1}{8 \alpha}\left(\frac{M}{M+1}\right)^{2} \frac{\log ^{2}\left(-\operatorname{Cov}\left(X_{p_{n}}, X_{2 p_{n}+1}\right)\right)}{\log n}, \\
& p_{n}=\frac{1}{2 x_{n}} \frac{n}{r_{n}}, 0<x_{n} \rightarrow 1
\end{aligned}
$$

and all one has to do is solve for $r_{n}$. Then, the corresponding rate of convergence is obtained from (3.31).

Remark 3.3. From (3.31), it follows that the optimal convergence rate is obtained by taking $r_{n}=n$. However, such a choice is not allowed here. Consequently, the convergence rate $(n / \log n)^{1 / 2}$ is unattainable in the present framework.

Remark 3.4. From (3.31), by taking $r_{n}=\sqrt{n}$ we have the convergence rate $(\sqrt{n} / \log n)^{1 / 2}$. 


\section{Examples}

In this section, two examples with common covariance functions are discussed. In each case, the optimal choice of $r_{n}$, provided by formula (3.34) is given as well as the corresponding best rate of almost sure convergence through formula (3.31).

Example 4.1. Suppose that $\operatorname{Cov}\left(X_{1}, X_{k+1}\right)=\rho_{0} \rho^{k}, 0<\rho<1, \rho_{0}<0$. Then relation (3.34) is of the form:

$$
r_{n}=C_{1 n} \frac{1}{\log n}+C_{2 n} \frac{n}{r_{n} \log n}+C_{3 n} \frac{n^{2}}{r_{n}^{2} \log n}
$$

or

$$
r_{n}{ }^{3}=C_{1 n} \frac{r_{n}^{2}}{\log n}+C_{2 n} \frac{n r_{n}}{\log n}+C_{3 n} \frac{n^{2}}{\log n},
$$

and the last term on the right-hand side in (4.1) is of highest order. Therefore $r_{n}{ }^{3}$ is of the order $n^{2} / \log n$ and $r_{n}$ is of the order $\left(n^{2} / \log n\right)^{1 / 3}$. Then, by (3.31), it turns out that $1 / \varepsilon_{n}$ is of the order $\left(n /(\log n)^{2}\right)^{1 / 3}$.

Example 4.2. Suppose $\operatorname{Cov}\left(X_{1}, X_{k+1}\right)=a_{0} k^{-\lambda}, \lambda>0, a_{0}<0$.

Then, we have

$$
\begin{aligned}
r_{n}= & C_{1 n} \frac{1}{\log n}+C_{2 n} \frac{\log r_{n}}{\log n}+C_{3 n} \frac{\left(\log r_{n}\right)^{2}}{\log n}+C_{4 n}+C_{5 n}\left(\log r_{n}\right) \\
& +C_{6 n}(\log n),
\end{aligned}
$$

and the last term on the right-hand side in (4.2) is of highest order. Thus, $r_{n}$ is of order $\log n$ and then, $1 / \varepsilon_{n}$ is a constant. Therefore, in this case, we do have almost sure convergence without rates.

\section{Acknowledgements}

The authors would like to thank the referee for his/her careful reading and useful comments that improved presentation of the manuscript.

\section{References}

[1] Block, H.W., Savits, T.H. and Sharked, M. (1982). Some concepts of negative dependence. Ann. Probab. 10 765-772. MR0659545

[2] Devroye, L. (1991). Exponential inequalities in nonparametric estimation. In: Roussas, G. (Ed.), Nonparametric Functional Estimation and Related Topics. Kluwer Academic Publishers, Dordrecht, pp. 31-44. MR1154318

[3] Ebrahimi, N. and Ghosh, M. (1981). Multivariate negative dependence. Comm. Statist. 10 307-337. MR0612400 
[4] Ionnnides, D.A. and Roussas, G.G. (1999). Exponential inequality for associated random variables. Statist. Probab. Letters 42 423-431. MR1707188

[5] Johg-Dev, K. and Proschan, F. (1983). Negative association of random variables, with application. Ann. Statist. 11 286-295. MR0684886

[6] JoAg-Dev, K. and Patil, G.P. (1975). Probability inequalities for certain multivariate discrete distributions. Sankhya 37 158-164. MR0652747

[7] Karlin, S. and Rinote, Y. (1980). Classes of orderings of measures and related correlation inequalities. II. Multivariate reverses rule distributions. J. multivar. Anal. 10 499-516. MR0599686

[8] Lehmann, E.L. (1966). Some concepts of dependence. Ann. Math. Statist. 37 1137-1153. MR0202228

[9] Oliveira, P.E. (2005). An exponential inequality for associated variables. Statist. Probab. Letters 73 189-197. MR2159254

[10] Newman, C.M. (1980). Normal fluctuation and the FKG systems. Comm. Math. Phys. 74 119-128. MR0576267 